\title{
Malnutrition and its risk among Portuguese older adults: Prevalence and underestimation using the short form of the Mini Nutritional Assessment
}

\author{
$\underline{\text { Rui Poínhos }}^{1}$, Bruno M. P. M. Oliveira ${ }^{1,2}$, Anzhela Sorokina ${ }^{3}$, Bela Franchini ${ }^{1}$, Cláudia Afonso ${ }^{1}$ \\ and Maria Daniel Vaz de Almeida ${ }^{1,4}$ \\ ${ }^{1}$ Faculdade de Ciências da Nutrição e Alimentação, Universidade do Porto, Porto, Portugal, \\ ${ }^{2}$ Laboratório de Inteligência Artificial e Apoio à Decisão, Instituto de Engenharia de Sistemas e Computadores - \\ Tecnologia e Ciência, Porto, Portugal, \\ ${ }^{3}$ Universidade do Porto (MSc student in Consumer Sciences and Nutrition), Porto, Portugal and \\ ${ }^{4}$ GreenUPorto - Centro de Investigação em Produção Agroalimentar Sustentável, Porto, Portugal
}

\begin{abstract}
Malnutrition is a serious health problem among older adults. The Mini Nutritional Assessment (MNA) is the most used tool to assess malnutrition among older adults. It was proposed that the Screening section could be used as a short form of the MNA (MNA-SF), but the study of the two forms show a wide range of agreement ( 71.8 to $93.3 \%$ and Cohen's kappa from 0.52 to 0.78 ). Nevertheless, most of the studies recommend the use of the MNA-SF, but in some this conclusion is not completely congruent with the results. The aims of this work were to study the agreement between the nutritional status classification using the MNA-SF and the MNA-FF among Portuguese older adults living in the community, to compare the scores in each question of the MNA-FF between participants correctly or incorrectly classified using the MNA-SF, and to develop a more sensible version of the MNA-SF. The current study is part of the PRONUTRISENIOR project. Data from 456 older adults $(54.2 \%$ females $)$ aged 65 to 92 years $($ mean $=73 ;$ SD $=6)$ living in the community (Vila Nova de Gaia, Portugal) were analyzed. The agreement between the two classifications is $82.7 \%$, but Cohen's $\mathrm{k}$ shows a weak agreement (weighted $\mathrm{k}=0.497 ; \mathrm{p}<0,001)$. MNA-SF showed a very low sensitivity $(42.6 \%)$ detecting participants malnourished or at risk of malnutrition. Participants classified as normal using the MNA-SF despite at risk using the MNA-SF presented lower scores in two items from the Assessment section: number of full meals eaten daily and amount of fluid consumed per day. These two items were included in MNA-SF to obtain an extended short-version (MNA-SF8), which presented a sensitivity of $91.8 \%$ (and a specificity of 79.9\%). The areas under the ROC curves were 0.858 and 0.929 , respectively for MNA-SF and MNA-SF8. The addition of two items to the MNA-SF provides a more sensible tool to detect risk of malnutrition among elderly. These findings suggest that, among the studied population, general eating-related questions regarding (full meals and fluid intake) are relevant to assess malnutrition. Similar studies should be carried out among other populations in order to verify the possibility of their generalization. [The PRONUTRISENIOR project was financed by Iceland, Liechtenstein and Norway through the EEA Grants (PT06 - Public Health Initiatives, reference 81NU5).]
\end{abstract}

\section{Conflict of Interest}

There is no conflict of interest. 